\title{
A hemifused complex is the hub in a network of pathways to membrane fusion
}

Jason M. Warner ${ }^{\mathrm{a}, \mathrm{b}}$, Dong An ${ }^{\mathrm{a}}$, Benjamin S. Stratton ${ }^{\mathrm{a}}$, Ben O'Shaughnessy ${ }^{\mathrm{a}, 1}$

a: Department of Chemical Engineering, Columbia University, New York, NY 10027

b: St. Luke's School, New Canaan, CT 06840

June 20, 2021

1: To whom correspondence may be addressed. Email: bo8@ columbia.edu 


\begin{abstract}
Membrane fusion is required for essential processes from neurotransmission to fertilization. For over 40 years protein-free fusion driven by calcium or other cationic species has provided a simplified model of biological fusion, but the mechanisms remain poorly understood. Cationmediated membrane fusion and permeation are essential in their own right to drug delivery strategies based on cell-penetrating peptides or cation-bearing lipid nanoparticles. Experimental studies suggest cations drive anionic membranes to a hemifused intermediate which serves as a hub for a network of pathways, but the pathway selection mechanism is unknown. Here we develop a mathematical model that identifies the network hub as a highly dynamical hemifusion complex. We find multivalent cations drive expansion of a high tension hemifusion interface between interacting vesicles during a brief transient. During this window, rupture of the interface competes with vesicle membrane rupture to determine the outcome, either fusion, dead-end hemifusion or vesicle lysis. The model reproduces the unexplained finding that fusion of vesicles with planar membranes typically stalls at hemifusion, and we show that the equilibrated hemifused state is a novel lens-shaped complex. Thus, membrane fusion kinetics follow a stochastic trajectory within a network of pathways, with outcome weightings set by fusogen concentration, vesicle size, lipid composition and geometry.
\end{abstract}

\title{
Significance
}

Cells use multicomponent machineries to fuse membranes for neurotransmitter and hormone release and other fundamental processes. Protein-free fusion using calcium or other multivalent cationic fusogens has long been studied as a simplifying model. Cation-mediated membrane fusion or permeation are key events for a number of current drug delivery strategies. However, the mechanisms determining outcomes are unknown. Here we develop a mathematical model that identifies a dynamic hemifusion complex as the decision hub that stochastically sets the outcome in a network of pathways. Cations transiently grow a high tension hemifusion interface 
bioRxiv preprint doi: https://doi.org/10.1101/2021.06.20.449175; this version posted June 20, 2021. The copyright holder for this preprint (which was not certified by peer review) is the author/funder. All rights reserved. No reuse allowed without permission.

between membrane-enclosed compartments, whose fate governs whether fusion, dead-end hemifusion or vesicle lysis occurs. The model provides a systematic framework to predict outcomes of cationic fusogen-mediated interactions between membrane-enclosed compartments. 


\section{Introduction}

Membrane fusion is essential for exocytosis, intracellular trafficking, fertilization and other processes vital for living organisms $(1,2)$. Fusion in cells is regulated by multicomponent cellular machineries, many aspects of which remain poorly understood $(3,4)$. For over 40 years protein-free fusion has served as a simplified model of biological fusion, retaining the complexities of phospholipid membranes but with simplified fusogens, most commonly calcium or other divalent cations (5-11). Protein-free systems could help elucidate the pathways to biological fusion and the biophysical mechanisms governing lipid bilayers that cellular fusion machineries must contend with over many scales, from $\sim 50$-nm synaptic vesicles to cortical granules with sizes from $~ 100 \mathrm{~nm}$ to several microns (12-17).

Cation-mediated fusion and membrane permeation also find use in a number of biotechnologies for delivery of drugs and other cargoes. Cationic cell-penetrating peptides (CPPs) with < 30 amino acids have been widely studied as agents of drug delivery for cancer, central nervous system diseases and others $(18,19)$, either covalently bonded to small cargo molecules or physically adhered to negatively charged cargoes such as DNA or RNA $(18,19)$. Examples of CPPs include a polybasic sequence from TAT, a transcription activator protein of HIV (20), and the model CPP nona-arginine (R9) (21). How cargoes are delivered is not established, but CPPs increase membrane permeability (22-25) and R9 was shown to fuse large vesicles (22). Similarly, antimicrobial peptides are naturally occurring and synthetically mimicked antibiotics, often positively charged, that permeabilize target membranes (26).

By far the most studied cationic fusogen is calcium. Historically calcium was proposed to be used by cells as a fusogen (27). Early in vitro studies in bulk suspension $(5-8,28)$ and later single event methods tracking $\mathrm{Ca}^{2+}$-mediated fusion of vesicles with planar bilayers (black lipid membranes, PMs) (29-31) identified several calcium-driven processes on the pathway to fusion. Adhesion of membranes was followed by hemifusion, a preliminary step fusing the outer monolayers only. The hemifused state was the final outcome ("dead-end hemifusion"), or 
hemifusion evolved into either full fusion or vesicle lysis, the rupture of a non-contacting membrane surface.

These discoveries suggest hemifusion is the critical intermediate on the fusion pathway. Indeed, in biological contexts hemifused intermediates are seen in live cells (16) and in vitro driven by SNARE proteins and other cellular fusion machinery components $(32,33)$, while hemifusion is observed in computer simulations $(34,35)$. Explicit support for hemifusion as the intermediate that sets the fusion outcome was provided by a study in which divalent cations adhered and hemifused giant unilamellar vesicles (GUVs) (11). At high calcium concentrations $\left(6 \mathrm{mM} \mathrm{Ca}^{2+}\right)$ the hemifused connections grew within seconds into micron sized hemifusion diaphragms (HDs) which ruptured to give fusion, or else vesicle lysis occurred. Lower concentrations produced dead-end hemifusion, with equilibrated HDs. A mathematical model $(36,37)$ reproducing the observed growth rates and HD sizes explained the HD expansion as driven by the powerful tendency of $\mathrm{Ca}^{2+}$ to contract anionic lipid monolayers and bilayers $(6,7$, $38,39)$ and dramatically increase the membrane tension as a result.

The picture from ref. (11) is that the pathway to membrane fusion is hemifusion followed by rupture of an expanding HD. Consistent with this pathway, $\mathrm{Ca}^{2+}$ drives dead-end hemifusion of GUVs with planar membranes (PMs), but fusion is triggered by osmotic boosting of the membrane tension or application of spontaneous curvature lysolipids to the distal PM monolayer (29-31). This suggests positive curvature pores expand to rupture the HD, but only if pore curvature energetics permit or if the membrane tension is sufficient. Negative curvature phospholipids such as PE have the opposite effect, as $\mathrm{Ca}^{2+}$ fuses or lyses pure PS GUVs, but lysis is abolished when PE is present $(9,10)$.

In summary, multivalent cations catalyse membrane fusion via a network of pathways passing through the hemifused state, but the pathway selection mechanism is unknown. Here we develop a mathematical model that predicts the pathway weightings and quantitatively reproduces the principal experimental findings. We show that, following nucleation, the HD has very high tension, but the HD grows and its tension decays. Adapting the measurements and 
model of ref. (40) to predict membrane rupture times, we find that depending on its tension decay the HD may rupture (fusion outcome) or survive the episode and equilibrate (dead-end hemifusion). A third possibility is vesicle membrane rupture, dependent on the tension history of the non-HD vesicle membranes (lysis outcome). The model reproduces the unexplained finding that, unless assisted by osmotic pressure or positive curvature lipids, fusion of vesicles with planar membranes typically stalls at hemifusion, and we show that this hemifused state is a novel lens-shaped complex. Thus, membrane fusion kinetics follow a stochastic trajectory within a network of pathways, with outcome weightings set by fusogen concentration, vesicle size, lipid composition and geometry.

\section{Results}

\section{Model}

We consider a pair of anionic lipid vesicles in the presence of $\mathrm{Ca}^{2+}$ (Fig. 1A). Calcium strongly adheres negatively charged or zwitterionic lipid vesicles, with adhesion energy $W$ per unit area dependent on cation concentration and lipid composition (41, 42). Experimentally, the strong adhesion catalyzes hemifusion of GUVs after $\sim 1-10 \mathrm{~s}(30,31,43)$ and nucleates a HD (11).

A second effect of $\mathrm{Ca}^{2+}$ is to boost membrane tension (e.g. $8 \mathrm{mN} / \mathrm{m}$ for DOPS monolayers with $\left.2 \mathrm{mM} \mathrm{Mg}^{2+}(6)\right)$, since $\mathrm{Ca}^{2+}$ tends to contract membranes by factors $\epsilon_{\text {cat }} \approx 5-$ $9 \%(6,7,38,39)$. This increased membrane tension drives HD growth, since tension favors smaller areas and a larger HD decreases the total area of the inner and outer leaflets in the hemifused complex $(36,37)$ (Fig. 1A). Further, $\mathrm{Ca}^{2+}$ selectively contracts the outer bilayer leaflets, also favoring HD growth.

Consider the vesicle membrane region. To grow the HD, the inner and outer leaflets of the non-HD vesicle membranes must slide relative to each other (Fig. S1). This produces a difference in the leaflet lipid densities $\Delta \rho$, which builds up interleaflet tension in that region,

$$
\gamma_{\Delta}=k_{\Delta}\left(\frac{\Delta \rho}{\rho_{0}}-\epsilon_{\text {cat }}\right)
$$


where the interleaflet modulus has typical values $k_{\Delta} \sim 20 \mathrm{mN} / \mathrm{m}(44)$, and $\rho_{0}$ is the initial density. Thus, calcium drives sliding of the vesicle membrane leaflets (and hence HD growth) since it favors a non-zero relative density difference, $\epsilon_{\mathrm{cat}}$.

The evolution of the leaflet density difference in the vesicle mebrane region is set by the balance between the force due to interleaflet tension and the interleaflet drag force, $\lambda \Delta \mathrm{v}=$ $-\nabla \gamma_{\Delta}$, where $\Delta \mathrm{v}$ is the difference in leaflet velocies and $\lambda$ the interleaflet friction coefficient. Using the continuity equation $\partial \Delta \rho / \partial t=-\rho_{0} \nabla \cdot(\Delta \mathbf{v})$ yields the density evolution dynamics

$$
\frac{\partial \Delta \rho}{\partial t}=\frac{k_{\Delta}}{\lambda} \nabla^{2}(\Delta \rho)
$$

These are to be solved subject to eq. (1) at the vesicle-HD boundary, relating $\Delta \rho$ to the interleaflet tension $\gamma_{\Delta}$. To convert this to a condition invoving the vesicle tension, we use the fundamental relation at this location between the $\mathrm{HD}$, vesicle and interleaflet tensions, $\gamma_{H D}=$ $\gamma_{\mathrm{ves}}+2 \gamma_{\Delta}$ (36). Further, the force balance at that location is $\gamma_{H D} \approx 2 \gamma_{\mathrm{ves}}$, valid for small contact angle $\theta$ (Fig. S1), yielding $\gamma_{\Delta} \approx \gamma_{\mathrm{ves}} / 2$. Using this in eq. (1) gives the boundary condition

$$
\left(\frac{\Delta \rho}{\rho_{0}}\right)_{r=R_{\mathrm{hd}}(t)}=\frac{\gamma_{\mathrm{ves}}(\mathrm{t})}{2 k_{\Delta}}+\epsilon_{\mathrm{cat}}
$$

where $R_{h d}(t)$ is the HD radius (the origin of coordinates is the HD center, $r=0$ ). Finally, the HD outer edge velocity $d R_{h d} / d t$ equals $\Delta \mathrm{v}$ at that location, equal to $-\nabla \gamma_{\Delta} / \lambda$. From eq. (1) this gives

$$
\frac{d R_{\mathrm{hd}}}{d t}=\frac{k_{\Delta}}{\lambda} \nabla\left(\frac{\Delta \rho}{\rho_{0}}\right)_{r=R_{\mathrm{hd}}(t)}
$$

In summary, our procedure is as follows $(36,37)$. We solve the density difference dynamics in the vesicle membrane region, eq. (2), subject to the moving boundary condition at the moving HD edge, eq. (3). Then eq. (4) gives the HD edge velocity, allowing continuous update of the boundary condition location. The solution yields the increasing HD size and area, $A_{\mathrm{HD}}(t)$. 
The HD expansion is driven by the vesicle membrane tension $\gamma_{\mathrm{ves}}(t)$ appearing in the boundary condition, eq. (3). Initially very high due to calcium, the tension decays with time due to three principal effects, which were all accounted for (see SI text for details). (i) As the HD grows, the total leaflet area (inner and outer combined) decreases. (ii) Slow water leakage on sec timescales decreases the vesicle volumes. (iii) The tension decrease due to (i) and (ii) is buffered somewhat by vesicle-vesicle adhesion, which flattens the vesicles and boosts tension.

Membrane rupture kinetics. This analysis yields the time-dependent areas and tensions of the HD and vesicle membranes. For high calcium concentrations, the tensions are sufficient to provoke rupture (see below). In ref. (40) higher tensions were shown experimentally to rupture membranes more rapidly. Adapting the mathematical model of tension-mediated rupture from that study, we arrive at the following kinetic scheme

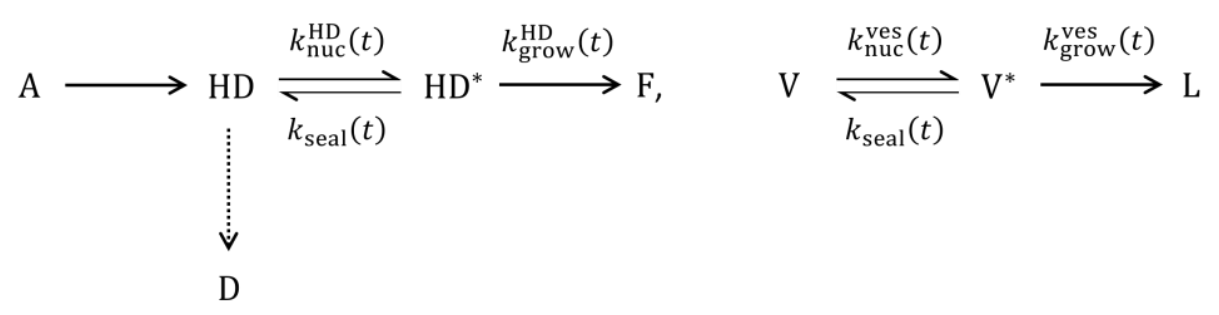

Here $\mathbf{A}$ is the initial tightly adhered vesicle state; HD and HD* are states with an expanding HD, with or without a nucleated pore in the HD, respectively; $\mathbf{F}$ is the fused state following pore growth and HD rupture (Fig. 1B). The second kinetic scheme describes simultaneous processes in the non-HD vesicle membranes: $\mathbf{V}$ and $\mathbf{V}^{*}$ denote vesicle membrane states with or without a pore, respectively, and $\mathbf{L}$ the lysis state. Finally, $\mathbf{D}$ denotes the dead end hemifused state.

As HD nucleation times have not been documented to our knowledge, for simplicity we assume this first step $\mathbf{A} \rightarrow$ HD is instantaneous (for the effects of finite HD nucleation times, see SI Fig. S4). The pore nucleation rate $k_{\text {nuc }}^{\mathrm{HD}}=\mathrm{A}_{\mathrm{hd}} r_{\mathrm{nuc}} \mathrm{e}^{\gamma_{\mathrm{hd}} / \gamma^{*}}$, and the rate constant for pore growth and HD lysis $k_{\text {grow }}^{\mathrm{HD}}=k_{\text {grow }}^{\mathrm{o}}\left(\gamma_{\mathrm{HD}} / \gamma_{\text {crit }}\right)^{1 / 2} \mathrm{e}^{-\gamma_{\text {crit }} / \gamma_{\mathrm{HD}}}$, depend on the time-dependent HD area and tension. Here $\gamma_{\text {crit }} \equiv \pi \tau^{2} / k_{\mathbf{B}} T$ where $\tau$ is the pore line tension (see SI text, eqs. 
$\mathrm{S} 17, \mathrm{~S} 18)(40,45)$. Analogous expressions for rate constants apply to the vesicle membrane lysis kinetics, but with the time-dependent vesicle area and tension.

Method of calculation. Table 1 lists the parameter values for each experimental system to which we applied our model. We determined the $\mathrm{Ca}^{2+-}$-induced contraction factor $\epsilon_{\text {cat }}$ and initial membrane tension from a composition weighted average of measured values for pure lipid species $(6,7,38,39)$. Pore line tension $\tau$ was computed from spontaneous curvatures of pure lipid species, and we extended an earlier model (46) to include curvature dependent lipid partitioning effects (see SI text). The line tension $\tau$ is a critical factor, as larger $\tau$ leads to slower pore growth rates $k_{\text {grow }}^{\mathrm{HD}}, k_{\text {grow }}^{\mathrm{ves}}$, and slower membrane rupture. Adhesion energies $W$ were inferred from experimentally reported contact angles. With these parameters, we evolved the HD and vesicle areas and tensions as described above, and used these in the coupled differential equations representing the kinetic scheme of eq. (5). The solutions yielded the fraction of events whose final outcome was fusion, dead-end hemifusion or lysis. For details, see SI text.

\section{Fusion is the domant outcome at intermediate cation concentrations}

The model predicts that cationic fusogens drive membranes along a network of pathways whose hub is the hemifused state (Fig. 1B). How does the outcome distribution depend on the concentration of fusogenic divalent cations? We applied the model to the conditions of the GUVGUV fusion experiments of ref. (11), accounting for the vesicle dimensions, phospholipid composition and adhesion energies (Table 1).

We calculated outcome distributions over a range of $\left[\mathrm{Ca}^{2+}\right]$ values. Three regimes emerged (Fig. 2A). (1) Low concentrations, $\left[\mathrm{Ca}^{2+}\right] \lesssim 2 \mathrm{mM}$. Following HD nucleation, membrane tension drives HD expansion, but the HD membrane tension is always below the rupture threshold (see below). With high probability the HD therefore expands without rupture to its final equilibrium low-tension state, i.e. dead-end hemifusion results with occasional fusion events. As the vesicle membrane tension is even lower, lysis is improbable. (2) Intermediate 
concentrations, $2 \mathrm{mM} \lesssim\left[\mathrm{Ca}^{2+}\right] \lesssim 10 \mathrm{mM}$. The initial HD tension now exceeds the rupture threshold, but the vesicle tension does not. Thus HD rupture (fusion outcome) is far more likely than vesicle rupture (lysis outcome). Dead-end hemifusion is rare. (3) High concentrations, $\left[\mathrm{Ca}^{2+}\right] \gtrsim 10 \mathrm{mM}$. All initial tensions exceed the rupture thresholds, but the vesicle has far greater area than does the HD, i.e. many more membrane locations for nucleation of pores that are the precursors of rupture. Thus, lysis is most probable.

\section{Fusion occurs during a limited time window in which HD tension is high}

Next we compared our model to the findings of ref. (11), where $\mathrm{Ca}^{2+}$-mediated interactions between GUVs were studied at two cation concentrations, and growing HDs were visualized from the instant of nucleation (see Table 1 for parameters). At the lower concentration, $2 \mathrm{mM}$ $\mathrm{Mg}^{2+}$, the model predicts these experiments lie near the low/intermediate regime boundary (Fig. 2A), with similar outcome probabilities for dead-end hemifusion (56\%) and fusion (43\%) but almost no lysis (Fig. 2B). This is consistent with ref. (11), where stable dead-end hemifusion events were reported for these conditions (no attempt was made to record fusion or lysis events). For $6 \mathrm{mM} \mathrm{Ca}^{2+}$, conditions lie deep within the intermediate regime and the model predicts mainly fusion $(61 \%)$, with roughly equal probabilities of dead-end hemifusion (18\%) or lysis (21\%) (Fig. 2B). This outcome distribution is consistent with the reported 2 fusion events and 1 lysis event (11), though the small number of observations precludes firm conclusions.

Fusion can only occur in a limited window of time as the HD grows and its tension decays. At the lower $2 \mathrm{mM}$ cation concentration the HD tension initially exceeds the rupture threshold $\gamma_{\text {rupture, }}$, where pore growth and resealing rates are equal ( $k_{\text {grow }}=k_{\text {seal }}$, eq. (5) and $S I$ text) (Fig. 2C). According to the kinetics of eq. (1), even a single nucleated pore will likely grow and rupture a membrane above this tension. Initially the HD area is too small to grow pores, but during a $\sim 7 \mathrm{~s}$ window the HD tension remains above the rupture threshold as the area increases, yielding a net $\sim 50 \%$ HD rupture probability (fusion outcome). Should the HD survive this 
episode, its tension decays below threshold so rupture is no longer possible (dead-end hemifusion). Almost no lysis occurs as the vesicle membrane tension is always below rupture threshold.

At the high $6 \mathrm{mM} \mathrm{Ca}^{2+}$ concentration, the initial HD tension is higher, giving a greater net fusion probability during the super-threshold window (Fig. 2D). Since the vesicle tension is higher, and due to the large vesicle area, lysis is now a significant outcome. The distribution of fusion times peaks later and is broader than the lysis time distribution (Fig. 2E). This is because the initial lysis rate is high due to the large vesicle membrane area, but its tension rapidly drops below the rupture threshold so that lysis ceases, whereas the HD needs time to grow sufficiently before significant pore nucleation occurs, but then maintains a super-rupture tension for $\sim 10 \mathrm{~s}$. The predicted timescales are consistent with the reported times of two fusion events $(0.75$ and $1.75 \mathrm{~s})$ and one lysis event (1.5 s) (11).

\section{Negative curvature lipids suppress vesicle lysis by lowering pore enlargement rates}

While the fusogen concentration sets the driving force for fusion, the phospholipid bilayer composition sets the susceptibility to that force. To study lipid composition effects we applied the model to the experiments of refs. $(9,10)$ where fusion of $\sim 10 \mu \mathrm{m}$ GUVs was studied in bulk suspension, with varying amounts of the phospholipids PS and PE which have positive and negative spontaneous curvatures, respectively. PS/PE compositions from pure PS to $75 \% \mathrm{PE}$ were studied. We assumed $5 \mathrm{mM} \mathrm{Ca}^{2+}$. A subtlety is that in the presence of $\mathrm{Ca}^{2+}$ the anionic lipid PS is thought to develop negative spontaneous curvature (Table S1).

For pure PS vesicles, the model predicts dead-end hemifusion is very rare (3\%), with substantial probabilities for fusion (37\%) and vesicle lysis (60\%) (Fig. 3A). Now the positive spontaneous curvature of PS gives a low rupture threshold tension, and on addition of $\mathrm{Ca}^{2+}$ both vesicle and HD tensions are super-threshold (Fig. 3C). Thus one might expect exclusively lysis, 
since the vesicle has far greater area. However, because the PS curvature switches to a negative pore-hating value in the presence of $\mathrm{Ca}^{2+}$, the vesicle has a much higher rupture threshold than the internal HD (Fig. 3C). This mechanism protects the vesicle from rupture, allowing significant fusion (Fig. 3E). These predictions agree qualitatively with the experimental outcomes ( 60\% fusion, $\sim 40 \%$ lysis, no hemifusion).

In contrast, with $75 \% \mathrm{PE}$ content all membranes have very high rupture thresholds as the fusion pore line tension $\tau$ is increased $\sim 2.5$-fold due to the large negative curvature of PE that disfavors growth of positive curvature fusion pores (see SI Text and Fig. S2). Thus dead-end hemifusion is dominant ( $88 \%$ ), with $12 \%$ fusion and almost no lysis due to the low vesicle tension. In the experiments no lysis was seen (10), in agreement with these predictions, but fusion and dead end occurred with equal probability, in contrast to the model predictions. We note that the model predicts equally probable fusion and dead end outcomes at a slightly lower PE content of $\sim 65 \%$ (Fig. 3B).

\section{Calcium mediated vesicle-planar membrane fusion stalls at hemifusion}

According to our model the vesicle-planar membrane fusion pathway has three outcomes from the hemifused intermediate (Fig 4). HD rupture leads to fusion and release of vesicle contents into the trans side of the planar membrane (PM); vesicle rupture leads to content release on the cis side of the PM; dead-end hemifusion results if all bilayer regions survive rupture. We solved the model (Eqs. (1)-(5) and see SI) using parameters for the experimental conditions of ref (30) (Table 1). Because the PM tension is relatively low, the HD tension exceeds the lysis threshold only briefly, $0.20 \mathrm{~s}$ (eq. S32) such that $<0.1 \%$ of vesicles fuse (eq. S35). Dead-end hemifusion is by far the most probable outcome predicted. This is consistent with the findings of refs (30, 31), where $\mathrm{Ca} 2+$ failed to fuse vesicles with PMs, which instead stalled at hemifusion.

In experiments, vesicle-PM fusion was rescued from dead-end hemifusion by later addition of lysolipids or osmotic agents. To understand the effects of these supplemental 
fusogens, we must first understand the properties of the dead-end hemifusion complex. In vesicle-vesicle hemifusion, it is known that outer leaflet compression and tension relaxation lead to an equilibrium HD which occupies $\sim<10 \%$ of the vesicle surface (36). The PM, however, is a symmetric lipid reservoir with zero interleaflet tension and fixed membrane tension $\gamma_{\mathrm{pm}}$. Thus the HD grows until encompassing half the area of the vesicle, adopting a lens configuration with contact angle $\theta$ (Fig. 4). Equilibrium is reached when the vesicle is sufficiently stretched in the plane of the PM that its tension satisfies the equilibrium force balance $\gamma_{\mathrm{PM}}=2 \gamma_{\mathrm{HD}} \cos \theta$, where the vesicle and HD tensions are equivalent by symmetry.

The tension might be expected to increase with an effective modulus equivalent to half the bilayer stretch modulus, because the inner leaflet undergoes a simple areal stretch at fixed mass. However, bilayer leaflets are co-dependent: as the inner leaflet is stretched and its density decreases, the outer vesicle leaflet draws extra lipids from the PM reservoir to compensate such that its density increases during the stretch (eq. S39, S42). This is driven by the membrane tension (modulus K) which tends to increase mean leaflet density but is opposed by the interleaflet tension (modulus $k_{\Delta}$ ) which tends to decrease the leaflet density difference. As a consequence, the effective stretch modulus for the vesicle involves both moduli, and the tension increases according to

$$
\gamma_{\mathrm{HD}}=\gamma_{\mathrm{pm}}+K_{\mathrm{eff}}\left(\mathrm{A}_{\mathrm{ves}} / A_{\mathrm{ves}}^{0}-1\right), \quad K_{\mathrm{eff}}=4 k_{\Delta} /\left(1+4 k_{\Delta} / K\right),
$$

where $A_{\text {ves }}$ is the vesicle area (HD and non-HD regions) and its initial value is $A_{\mathrm{ves}}^{0}$. To leading order in the relative angle $\phi=90^{\circ}-\theta$ at constant vesicle volume, the vesicle area is $A_{\text {ves }}=A_{\text {ves }}^{0}\left(1+\phi^{2} / 4\right)$. Using this relationship in eq. (6) with the equilibrium force balance above, we solved for the equilibrium HD tension and contact angle,

$$
\gamma_{\mathrm{hd}}=b K_{\mathrm{eff}}^{1 / 3} \gamma_{\mathrm{pm}}^{2 / 3}, \theta=90^{\circ}+d\left(\frac{\gamma_{\mathrm{pm}}}{K_{\mathrm{eff}}}\right)^{1 / 3}
$$

where $b=0.40$ and $d=72^{\circ}$. Using the parameter values of Table 1 in eq. (7), the equilibrium HD tension is $2.6 \mathrm{mN} / \mathrm{m}$, safely below the lysis threshold of $8.4 \mathrm{mN} / \mathrm{m}$. 
The function of lysolipids in rescuing fusion after hemifusion may be to lower the lysis threshold below the equilibrium HD tension. According to the model, lysolipids added to the trans side of the PM will partition into the HD from the aqueous phase, and their positive spontaneous curvature depresses the pore line tension and thus the lysis tension. We find that the lysis threshold is lowered to that of the equilibrium HD tension, $2.6 \mathrm{mN} / \mathrm{m}$ for ref. (30) parameters, once the molar composition of LPC in the HD reaches $20 \%$. Using the partitioning data of ref. (47), the $270 \mu \mathrm{M}$ LPC solution used in the fusion studies of ref. $(30,31,48)$, for example, is more than sufficient to achieve $20 \%$ LPC in the HD, and hence drive fusion by lowering the lysis threshold.

The function of osmotic agents added after hemifusion may be to swell the vesicle to increase the HD tension beyond the lysis threshold. Since vesicle volume must adjust, the constant volume result of eq. (7) for HD tension is no longer valid. The simplest approach to calculating the HD tension uses the fact that the radius of HD curvature is essentially that of the vesicle, $R_{\text {ves }}$. Hence according to the Young-Laplace equation the HD tension is $\gamma_{\mathrm{hd}}=$ $P_{\mathrm{osm}} R_{\mathrm{ves}} / 2$, where $P_{\mathrm{osm}}$ is the osmotic pressure. Using the parameters of ref. (30), we thus find that the HD tension reaches its threshold value of $8.4 \mathrm{mN} / \mathrm{m}$ when the osmotic pressure reaches $3.4 \mathrm{kPa}$, corresponding to a threshold osmolarity difference of $1.3 \mathrm{mOsm} / \mathrm{kg}$. Thus the 75 $\mathrm{mOsm} / \mathrm{kg}$ osmolarity difference imposed in ref. (30) was sufficient to drive fusion by increasing the HD tension above the lysis threshold.

\section{Discussion}

\section{Calcium-mediated fusion is described by a network of pathways that pass through the hemifused intermediate.}

Here we showed that calcium-mediated fusion follows a network of pathways whose branch point is the hemifused intermediate (Fig 1B). The network is unchanging, but the conditions $\left(\mathrm{Ca}^{2+}\right.$ concentration, lipid composition, membrane areas, geometry) regulate the frequency with 
which different pathways are selected (Figs 2A, 3B, 4). Pathway selection is made at the hemifused branch point and consists in selecting which membrane surface first ruptures by nucleation and dilation of a pore governed by Evans kinetics model (40). Since the many-lipid pore dynamics are stochastic, the sampling of pathways in the network is stochastic, but the averages are fixed for a given set of conditions.

The fusion mechanism stems from powerful adhesion and contraction forces that divalent cations exert on charged membranes. These forces generate high membrane tensions and nucleate an expanding HD, a critical transition that channels two membrane tensions through the one HD bilayer (Fig. 1B, inset). However, even if the HD tension is high its rupture is not guaranteed because membranes can withstand super-rupture tensions for limited time periods (40). Since HD expansion progressively decreases tension, there is a small window in time when the HD may rupture (fusion); if the HD survives this transient, it grows to equilibrium (dead-end hemifusion, Figs. 2C, D and 3C, D). Another factor is membrane area, on which pore nucleation rates depend: the HD starts from a collapsed state and must grow before rupture is likely, while the vesicle area is much greater, so the vesicle may rupture first (lysis) despite its lower tension.

\section{Calcium concentration, lipid composition and membrane areas set the weightings of pathways in the network.}

We found that increasing $\mathrm{Ca}^{2+}$ concentration increases membrane tensions and rupture probability, so reducing the weighting for dead-end hemifusion (Fig. 2A, B). However the balance between fusion and lysis is more subtle since area comes into play: when all membranes are at super-rupture tensions, the larger vesicle membrane is more likely to rupture (lysis) as it nucleates pores more quickly. Accordingly, fusion has maximum weighting at intermediate $\left[\mathrm{Ca}^{2+}\right]$ and tension (Fig. 2A). Interestingly, fusion was also maximized at intermediate $\left[\mathrm{Ca}^{2+}\right]$ in bulk suspensions of small vesicles (6). Similarly, lipid composition radically influences the pathway weightings by altering the line tension of positive curvature membrane pores; for 
example, increased content of PE, a negative curvature lipid, increased the line tension, and the energy cost of pore expansion, with lowered fusion probability (Fig. 3).

\section{The effect of membrane geometry: calcium is ineffective at vesicle-planar membrane fusion.}

$\mathrm{Ca}^{2+}$-driven fusion assays using the vesicle-planar membrane geometry that arises during exocytosis are of particular interest. Surprisingly, calcium is an ineffective fusogen with this geometry $(30,31,48)$, an unexplained fact. With typical experimental conditions our model reproduced the observed dead-end hemifusion: tensions dissipate too rapidly for fusion to occur, and the final state is an equilibrium lens-shaped low tension hemifused complex (Fig. 4).

Why is $\mathrm{Ca}^{2+}$ so effective at fusing vesicles (Figs. 2-3), yet cannot (without help from osmotic stress or lysolipids) fuse vesicles with a planar PM (Fig. 4)? There are two key factors. (i) PM tensions are not elevated by $\mathrm{Ca}^{2+}$-induced contraction, because the PM can draw lipids from the reservoir at its supporting torus to dissipate tension. Thus the initial HD tension is much lower than in vesicle-vesicle systems, where the HD supports two $\mathrm{Ca}^{2+}$-boosted vesicle tensions (Fig. 1, inset). (ii) The macroscopic PM is an effectively infinite lipid reservoir (49) unaffected by the hemifusion event. Thus, large $\mathrm{Ca}^{2+}$-induced vesicle tensions are dissipated on hemifusion with the PM, and we showed that the final equilibrium HD tension depends only on the relatively low tension of the PM (see eq. (4)).

\section{Biological and calcium-mediated fusion may follow similar pathway networks.}

$\mathrm{Ca}^{2+}$-mediated fusion has long been studied as a protein-free model of biological fusion. While $\mathrm{Ca}^{2+}$ fuses membranes through globally-acting membrane tension, the cell's fusion machinery appears to act in a relatively small zone at the contact point of two membranes. Tension was proposed to regulate kiss-and-run fusion/promote exocytotic fusion of secretory granules in human neuroendocrine cells (50) and enlarged pores in a hemagglutinin mediated fusion system (51), but the extent of its role is uncertain. 
Nonetheless, biological fusion may follow a network of fusion pathways centered on the hemifused intermediate similar to that for $\mathrm{Ca}^{2+}$-mediated fusion (Fig. 1B). Long-lived hemifused intermediates were detected on the fusion pathway in pneumatocytes and during yeast vacuole fusion $(52,53)$. Electron microscopy revealed extended HDs between synaptic vesicles and the plasma membrane ( $\sim 5 \mathrm{~nm}$ in diameter), between granules and the plasma membrane of chromaffin cells $(\sim 200 \mathrm{~nm})$ and between yeast vacuoles $(\sim 0.5-1 \mu \mathrm{m})(12,13,16,54)$.

Thus, some biological fusion pathways may pass through hemifused intermediates with extended HDs. This is supported by data from reconstituted systems. In a study of fusion mediated by SNARE and synaptotagmin proteins, docked vesicle pairs either dead-end hemifused or remained docked $(32,33)$. Upon introduction of $\mathrm{Ca}^{2+}$ the docked vesicles remained docked, fused via a hemifused intermediate, fused directly or dead-end hemifused. Thus, higher fusion driving force $\left(\mathrm{Ca}^{2+}\right.$ present) increases the fusion probability, similar to the pattern for $\mathrm{Ca}^{2+}$ driven fusion, Fig. 2A, B. Similarly, SNARE-mediated fusion rates were lower and dead-end hemifusion more probable for lower vesicle SNARE density (55) or when SNARE zippering was impeded by either hydrophobic molecules (56) or a mutation (57). Hernandez et al. (32) report that vesicles docked by SNAREs in point or extended contact adhered states evolved to dead-end hemifusion or fusion (32). The presence of PE in vesicle membranes reduced the incidence of SNARE-mediated fusion of vesicles with supported bilayers, produced significant dead-end hemifusion and decreased the hemifusion to fusion transition rate (58), similar to our predictions for the pore growth rate $k_{\text {grow }}$ (eq. (5) and Fig. 3). Finally, hemifusion is a productive intermediate on the pathway to fusion mediated by influenza's fusion protein hemagglutinin, and can also be a dead-end state (59-61). More interestingly, hemifused intermediates were observed in chromaffin cells (16).

These parallels suggest that the network of fusion pathways may be intrinsic to the lipid bilayers themselves, independently of the fusogen involved. Different fusogens may generate different outcome distributions and pathway kinetics due to their differing force-generating mechanisms. Although SNAREs, hemagglutinin and calcium each likely exhibit unique aspects to their force 
generation mechanisms, they still must contend with the same resisting forces against fusion pathway progress, set by the physical properties of the lipids bilayers, examined in detail in this work.

\section{Acknowledgements}

Research reported in this publication was supported by the National Institute of General Medical Sciences of the National Institutes of Health under award number R01GM117046 to B.O. The content is solely the responsibility of the authors and does not necessarily represent the official views of the National Institutes of Health. We thank Andreas Hermann and Joerg Nikolaus for helpful discussions and access to their data prior to submission. 


\section{References}

1. S. Martens, H. T. McMahon, Mechanisms of membrane fusion: disparate players and common principles. Nat Rev Mol Cell Biol 9, 543-556 (2008).

2. W. Wickner, R. Schekman, Membrane fusion. Nat Struct Mol Biol 15, 658-664 (2008).

3. A. T. Brunger, U. B. Choi, Y. Lai, J. Leitz, Q. Zhou, Molecular Mechanisms of Fast Neurotransmitter Release. Annu Rev Biophys 47, 469-497 (2018).

4. T. C. Sudhof, Neurotransmitter release: the last millisecond in the life of a synaptic vesicle. Neuron 80, 675-690 (2013).

5. L. Ginsberg, Does Ca2+ cause fusion or lysis of unilamellar lipid vesicles? Nature 275, 758-760 (1978).

6. S. Ohki, A mechanism of divalent ion-induced phosphatidylserine membrane fusion. Biochim Biophys Acta 689, 1-11 (1982).

7. S. Ohki, H. Ohshima, Divalent cation-induced phosphatidic acid membrane fusion. Effect of ion binding and membrane surface tension. Biochim Biophys Acta 812, 147-154 (1985).

8. J. Wilschut, D. Papahadjopoulos, Ca2+-induced fusion of phospholipid vesicles monitored by mixing of aqueous contents. Nature 281, 690-692 (1979).

9. R. P. Rand, B. Kachar, T. S. Reese, Dynamic Morphology of Calcium-Induced Interactions between Phosphatidylserine Vesicles. Biophysical Journal 47, 483-489 (1985).

10. B. Kachar, N. Fuller, R. P. Rand, Morphological responses to calcium-induced interaction of phosphatidylserine-containing vesicles. Biophys J 50, 779-788 (1986).

11. J. Nikolaus, M. Stockl, D. Langosch, R. Volkmer, A. Herrmann, Direct visualization of large and protein-free hemifusion diaphragms. Biophys J 98, 1192-1199 (2010).

12. G. A. Zampighi et al., Conical tomography of a ribbon synapse: structural evidence for vesicle fusion. PLoS One 6, e16944 (2011).

13. J. H. Jung, J. A. Szule, R. M. Marshall, U. J. McMahan, Variable priming of a docked synaptic vesicle. Proc Natl Acad Sci U S A 113, E1098-1107 (2016).

14. G. A. Perkins, D. R. Jackson, G. A. Spirou, Resolving presynaptic structure by electron tomography. Synapse 69, 268-282 (2015).

15. A. J. B. Kreutzberger et al., Distinct insulin granule subpopulations implicated in the secretory pathology of diabetes types 1 and 2. Elife $\mathbf{9}$ (2020).

16. W. D. Zhao et al., Hemi-fused structure mediates and controls fusion and fission in live cells. Nature 534, 548-552 (2016).

17. T. Rousso, E. D. Schejter, B. Z. Shilo, Orchestrated content release from Drosophila glueprotein vesicles by a contractile actomyosin network. Nat Cell Biol 18, 181-190 (2016).

18. J. Xie et al., Cell-Penetrating Peptides in Diagnosis and Treatment of Human Diseases: From Preclinical Research to Clinical Application. Front Pharmacol 11, 697 (2020).

19. J. Xu et al., Cell-penetrating peptide: a means of breaking through the physiological barriers of different tissues and organs. J Control Release 309, 106-124 (2019).

20. S. R. Schwarze, A. Ho, A. Vocero-Akbani, S. F. Dowdy, In vivo protein transduction: delivery of a biologically active protein into the mouse. Science 285, 1569-1572 (1999). 
21. K. Melikov et al., Efficient entry of cell-penetrating peptide nona-arginine into adherent cells involves a transient increase in intracellular calcium. Biochem J 471, 221-230 (2015).

22. C. Allolio et al., Arginine-rich cell-penetrating peptides induce membrane multilamellarity and subsequently enter via formation of a fusion pore. Proc Natl Acad Sci U S A 115, 11923-11928 (2018).

23. S. Sharmin et al., Effects of Lipid Composition on the Entry of Cell-Penetrating Peptide Oligoarginine into Single Vesicles. Biochemistry-Us 55, 4154-4165 (2016).

24. E. Trofimenko et al., Genetic, cellular and structural characterization of the membrane potential-dependent cell-penetrating peptide translocation pore. bioRxiv, 2020.2002. 2025.963017 (2021).

25. M. Serulla et al., TAT-RasGAP317-326 kills cells by targeting inner-leaflet-enriched phospholipids. Proc Natl Acad Sci U S A 117, 31871-31881 (2020).

26. M. Mahlapuu, J. Hakansson, L. Ringstad, C. Bjorn, Antimicrobial Peptides: An Emerging Category of Therapeutic Agents. Front Cell Infect Microbiol 6, 194 (2016).

27. P. R. Cullis, M. J. Hope, Effects of fusogenic agent on membrane structure of erythrocyte ghosts and the mechanism of membrane fusion. Nature 271, 672-674 (1978).

28. J. Bentz, H. Ellens, M. Z. Lai, F. C. Szoka, On the Correlation between Hii Phase and the Contact-Induced Destabilization of "Phosphatidylethanolamine-Containing Membranes. Proceedings of the National Academy of Sciences of the United States of America 82, 57425745 (1985).

29. M. H. Akabas, F. S. Cohen, A. Finkelstein, Separation of the osmotically driven fusion event from vesicle-planar membrane attachment in a model system for exocytosis. $J$ Cell Biol 98, 1063-1071 (1984).

30. L. Chernomordik, A. Chanturiya, J. Green, J. Zimmerberg, The hemifusion intermediate and its conversion to complete fusion: regulation by membrane composition. Biophys $J \mathbf{6 9}$, 922-929 (1995).

31. A. Chanturiya, L. V. Chernomordik, J. Zimmerberg, Flickering fusion pores comparable with initial exocytotic pores occur in protein-free phospholipid bilayers. Proc Natl Acad Sci U S A 94, 14423-14428 (1997).

32. J. M. Hernandez et al., Membrane fusion intermediates via directional and full assembly of the SNARE complex. Science 336, 1581-1584 (2012).

33. J. Diao et al., Synaptic proteins promote calcium-triggered fast transition from point contact to full fusion. Elife 1, e00109 (2012).

34. H. J. Risselada, G. Bubnis, H. Grubmuller, Expansion of the fusion stalk and its implication for biological membrane fusion. Proc Natl Acad Sci U S A 111, 11043-11048 (2014).

35. S. Sharma, M. Lindau, Molecular mechanism of fusion pore formation driven by the neuronal SNARE complex. Proc Natl Acad Sci U S A 115, 12751-12756 (2018).

36. J. M. Warner, B. O'Shaughnessy, The hemifused state on the pathway to membrane fusion. Phys Rev Lett 108, 178101 (2012).

37. J. M. Warner, B. O'Shaughnessy, Evolution of the hemifused intermediate on the pathway to membrane fusion. Biophys J 103, 689-701 (2012). 
38. D. Uhrikova, N. Kucerka, J. Teixeira, V. Gordeliy, P. Balgavy, Structural changes in dipalmitoylphosphatidylcholine bilayer promoted by $\mathrm{Ca} 2+$ ions: a small-angle neutron scattering study. Chem Phys Lipids 155, 80-89 (2008).

39. P. T. Vernier, M. J. Ziegler, R. Dimova, Calcium binding and head group dipole angle in phosphatidylserine-phosphatidylcholine bilayers. Langmuir 25, 1020-1027 (2009).

40. E. Evans, V. Heinrich, F. Ludwig, W. Rawicz, Dynamic tension spectroscopy and strength of biomembranes. Biophys J 85, 2342-2350 (2003).

41. K. Komorowski et al., Vesicle Adhesion and Fusion Studied by Small-Angle X-Ray Scattering. Biophys J 114, 1908-1920 (2018).

42. K. Akashi, H. Miyata, H. Itoh, K. Kinosita, Jr., Formation of giant liposomes promoted by divalent cations: critical role of electrostatic repulsion. Biophys J 74, 2973-2982 (1998).

43. G. Lei, R. C. MacDonald, Lipid bilayer vesicle fusion: intermediates captured by highspeed microfluorescence spectroscopy. Biophys J 85, 1585-1599 (2003).

44. R. E. Waugh, J. Song, S. Svetina, B. Zeks, Local and nonlocal curvature elasticity in bilayer membranes by tether formation from lecithin vesicles. Biophys J 61, 974-982 (1992).

45. J. D. Litster, Stability of Lipid Bilayers and Red Blood-Cell Membranes. Physics Letters A A 53, 193-194 (1975).

46. Y. A. Chizmadzhev, P. I. Kuzmin, D. A. Kumenko, J. Zimmerberg, F. S. Cohen, Dynamics of fusion pores connecting membranes of different tensions. Biophys J 78, 2241-2256 (2000).

47. D. Needham, D. V. Zhelev, Lysolipid exchange with lipid vesicle membranes. Ann Biomed Eng 23, 287-298 (1995).

48. F. S. Cohen, J. Zimmerberg, A. Finkelstein, Fusion of phospholipid vesicles with planar phospholipid bilayer membranes. II. Incorporation of a vesicular membrane marker into the planar membrane. J Gen Physiol 75, 251-270 (1980).

49. A. Chanturiya, P. Scaria, M. C. Woodle, The role of membrane lateral tension in calciuminduced membrane fusion. J Membr Biol 176, 67-75 (2000).

50. M. Bretou et al., Cdc42 controls the dilation of the exocytotic fusion pore by regulating membrane tension. Mol Biol Cell 25, 3195-3209 (2014).

51. R. M. Markosyan, G. B. Melikyan, F. S. Cohen, Tension of membranes expressing the hemagglutinin of influenza virus inhibits fusion. Biophys $J$ 77, 943-952 (1999).

52. P. Miklavc et al., Existence of exocytotic hemifusion intermediates with a lifetime of up to seconds in type II pneumocytes. Biochem J 424, 7-14 (2009).

53. C. Reese, F. Heise, A. Mayer, Trans-SNARE pairing can precede a hemifusion intermediate in intracellular membrane fusion. Nature 436, 410-414 (2005).

54. S. Mattie, E. K. McNally, M. A. Karim, H. Vali, C. L. Brett, How and why intralumenal membrane fragments form during vacuolar lysosome fusion. Mol Biol Cell 28, 309-321 (2017).

55. Y. Yang et al., Dissection of SNARE-driven membrane fusion and neuroexocytosis by wedging small hydrophobic molecules into the SNARE zipper. Proceedings of the National Academy of Sciences of the United States of America 107, 22145-22150 (2010). 
56. Y. Xu, F. Zhang, Z. Su, J. A. McNew, Y. K. Shin, Hemifusion in SNARE-mediated membrane fusion. Nat Struct Mol Biol 12, 417-422 (2005).

57. M. D'Agostino, H. J. Risselada, L. J. Endter, V. Comte-Miserez, A. Mayer, SNAREmediated membrane fusion arrests at pore expansion to regulate the volume of an organelle. EMBO J 37 (2018).

58. T. Liu, T. Wang, E. R. Chapman, J. C. Weisshaar, Productive hemifusion intermediates in fast vesicle fusion driven by neuronal SNAREs. Biophys J 94, 1303-1314 (2008).

59. T. Ivanovic, J. L. Choi, S. P. Whelan, A. M. van Oijen, S. C. Harrison, Influenza-virus membrane fusion by cooperative fold-back of stochastically induced hemagglutinin intermediates. Elife 2, e00333 (2013).

60. P. Chlanda et al., The hemifusion structure induced by influenza virus haemagglutinin is determined by physical properties of the target membranes. Nat Microbiol 1, 16050 (2016).

61. L. J. Calder, P. B. Rosenthal, Cryomicroscopy provides structural snapshots of influenza virus membrane fusion. Nat Struct Mol Biol 23, 853-858 (2016). 


\section{Table Captions}

\begin{tabular}{|c|c|c|c|c|c|c|c|c|c|}
\hline \multirow[b]{2}{*}{ System } & \multirow[b]{2}{*}{ Ref. } & \multicolumn{3}{|c|}{ Experimental Conditions } & \multicolumn{5}{|c|}{ Model Parameter Values } \\
\hline & & $\begin{array}{l}R_{\text {ves }} \\
(\mu m)\end{array}$ & [Cation] & Composition & $\begin{array}{c}\gamma^{0} \\
(\mathrm{mN} / \mathrm{m})\end{array}$ & $\begin{array}{l}\epsilon_{\text {cat }} \\
(\%)\end{array}$ & $\begin{array}{c}\mathbf{W} \\
(\mathrm{mN} / \mathbf{m})\end{array}$ & $\begin{array}{c}\tau \\
(\mathbf{p N})\end{array}$ & $\begin{array}{c}\gamma_{\text {crit }} \\
(\mathbf{m N} / \mathbf{m})\end{array}$ \\
\hline GUV-GUV & (11) & 9 & $6 \mathrm{mM} \mathrm{Ca}^{2+}$ & 60 PC/20 PS/20 PE & 8.6 & 6.3 & 1.1 & 12.8 & 125 \\
\hline GUV-GUV & (11) & 9 & $2 \mathrm{mM} \mathrm{Mg}^{2+}$ & $60 \mathrm{PC} / 20 \mathrm{PS} / 20 \mathrm{PE}$ & 7.2 & 5 & 1.1 & 12.8 & 125 \\
\hline GUV-GUV & $(9)$ & 5 & $5 \mathrm{mM} \mathrm{Ca}^{2+}$ & 100 PS & 9.4 & 7 & 5.3 & 12.6 & 122 \\
\hline GUV-GUV & (10) & 5 & $5 \mathrm{mM} \mathrm{Ca}^{2+}$ & $25 \mathrm{PS} / 75 \mathrm{PE}$ & 8.8 & 6.7 & 5.3 & 16.8 & 215 \\
\hline GUV-PM & (30) & 5 & $20 \mathrm{mM} \mathrm{Ca}^{2+}$ & $80 \mathrm{Aso} / 20 \mathrm{LPC}$ & 12.2 & 9.2 & - & 7 & 40 \\
\hline GUV-PM & $(30)$ & 5 & $20 \mathrm{mM} \mathrm{Ca}^{2+}$ & 100 Aso & 12.2 & 9.2 & - & 12 & 114 \\
\hline GUV-PM & $(31)$ & 5 & $20 \mathrm{mM} \mathrm{Ca}^{2+}$ & $88 \mathrm{Aso} / 12 \mathrm{Erg}$ & 10 & 7.5 & - & 13 & 127 \\
\hline
\end{tabular}

Table 1- Model parameters. Each line corresponds to one experiment to which the model was applied. Line tension $\tau$ and $\mathrm{Ca}^{2+}$-induced initial tensions $\gamma^{0}$ and cation contraction factors $\epsilon_{\text {cat }}$ were calculated from single species properties and the lipid composition (see SI text). Adhesion energies $W$ were estimated from the observed vesicle-substrate (11) or vesicle-vesicle $(9,10)$ contact angles (see SI Text). Pore kinetics rate parameters are taken as reported in ref. (40), based on rupture measurements of pure DOPC vesicles: $\gamma^{*}=4.0 \mathrm{mN} / \mathrm{m} ; r_{\text {nuc }}=1.8 \times 10^{-4} \mu \mathrm{m}^{-2} \mathrm{~s}^{-1}$; $k_{\text {grow }}^{\mathrm{o}}=3 \times 10^{6} \mathrm{~s}^{-1} ; k_{\text {seal }}=0.22 \mathrm{~s}^{-1} .$. Lipid abbreviations: PC: phosphocholine; PS:

phosphoserine; PE: phosphoethanolamine; LPC: lysophosphatidylcholine; Aso: asolectin; Erg: ergosterol. 


\section{Figures}

A

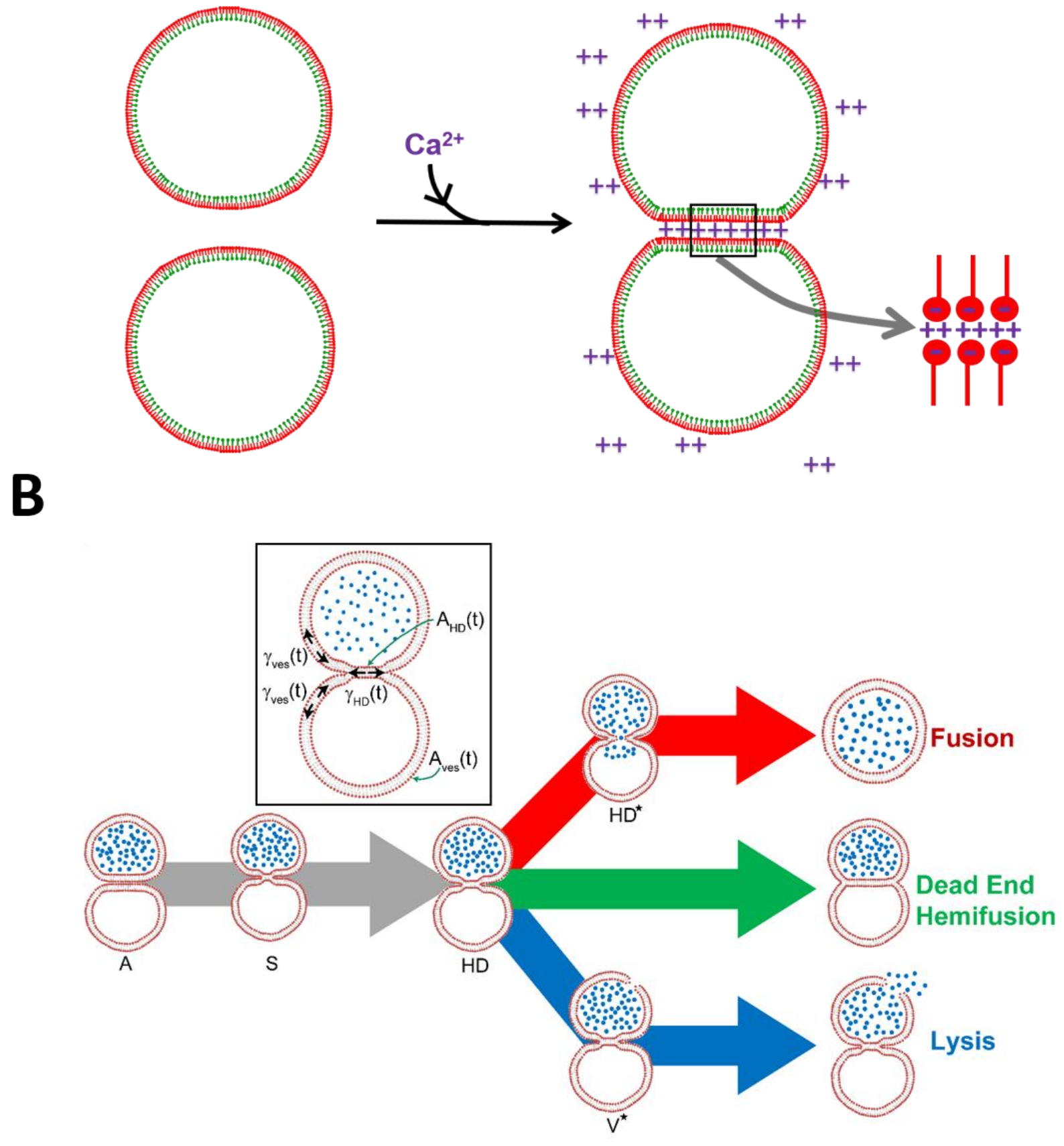


Figure 1- (A) $\mathrm{Ca}^{2+}$ increases membrane tension and adheres negatively charged vesicles. $\mathrm{Ca}^{2+}$ interacts with anionic or zwitterionic lipids in the outer leaflet of the vesicle membrane to contract the vesicle membrane by a factor $\epsilon$ to increase tension. A second effect is to adhere the vesicles, with adhesion energy $W$ per unit area. (B) Network of pathways to $\mathrm{Ca}^{2+}$-mediated membrane fusion. Calcium and other divalent cations adhere phospholipid bilayer vesicle membranes (state $\mathbf{A}$ ) and provoke hemifusion, fusion of the outer phospholipid monolayers only. The initial hemifusion connection is thought to be a minimal stalk (state $\mathbf{S}$ ) that is metastable and yields to an expanding hemifusion diaphragm, HD (state HD). HD expansion is driven by high calcium-induced membrane tension and outer monolayer contraction. The HD bilayer tension is greatest and may generate a pore (state HD*) that could reseal or dilate and rupture the HD (fusion outcome); or the vesicle membrane could nucleate a pore (state $\mathbf{V}^{*}$ ) that dilates and causes rupture (lysis outcome); or the HD may survive the high tension transient unscathed, expanding to full equilibrium (dead-end hemifusion outcome). Inset: The transient hemifused state (HD) selects the pathway. The HD tension $\gamma_{\mathrm{HD}}(t)$ is greatest, as it balances two vesicle tensions $\gamma_{\mathrm{ves}}(\mathrm{t})$, but its area is least, $A_{\mathrm{HD}}(t)<A_{\mathrm{ves}}(t)$. These effects compete to set the ratio of fusion to lysis. Since tension rapidly decays as the HD expands, the HD may escape to low tension equilibrium (dead-end hemifusion). 
A

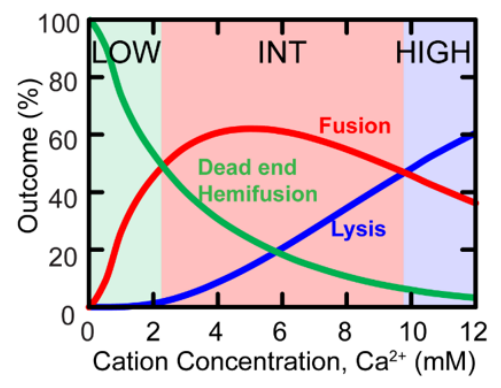

B

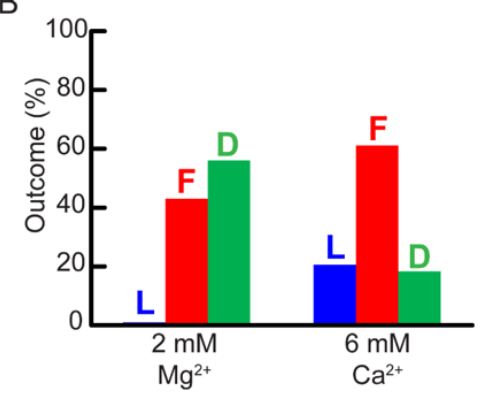

C

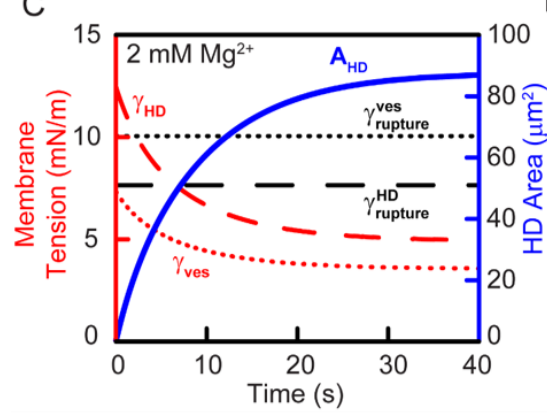

D
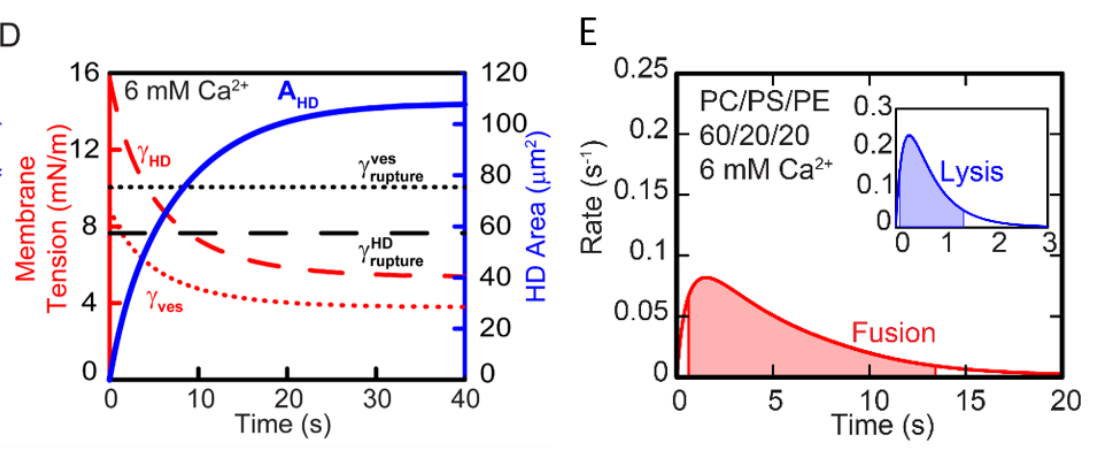

Figure 2- Calcium concentration governs outcome distribution in $\mathrm{Ca}^{2+}$-mediated GUV-GUV fusion. Model predictions, conditions as in experiments of ref. (11) (see Table 1 for parameters). GUVs are made of phospholipid with PS/PE/PC $=1 / 1 / 3$. (A) Predicted outcome distributions versus calcium concentration. In the low concentration regime dead-end hemifusion is most probable; fusion is maximized in the intermediate regime; lysis dominates at high concentrations. (B) Predicted outcome distributions for the two specific cation concentrations used in ref. (11). D, dead-end hemifusion; L, Lysis; F, fusion. (C, D) Predicted time evolution of HD area and tension and vesicle tension at $2 \mathrm{mM} \mathrm{Mg}^{2+}(\mathrm{C})$ and $6 \mathrm{mM} \mathrm{Ca}^{2+}(\mathrm{D})$. HD growth decays the vesicle and HD tensions to below their respective rupture thresholds. More fusion occurs at the higher concentration because the HD tension exceeds its rupture threshold for longer. Calcium only interacts with the PS component in the non-HD region to switch its spontaneous curvature to a negative pore-hating value. Compared with HDs, non-HD membranes are less likely to rupture. (E) Model predicted rates of $\mathrm{Ca}^{2+}$-mediated GUV-GUV fusion and lysis versus time for the conditions of the experiments of refs (11). 

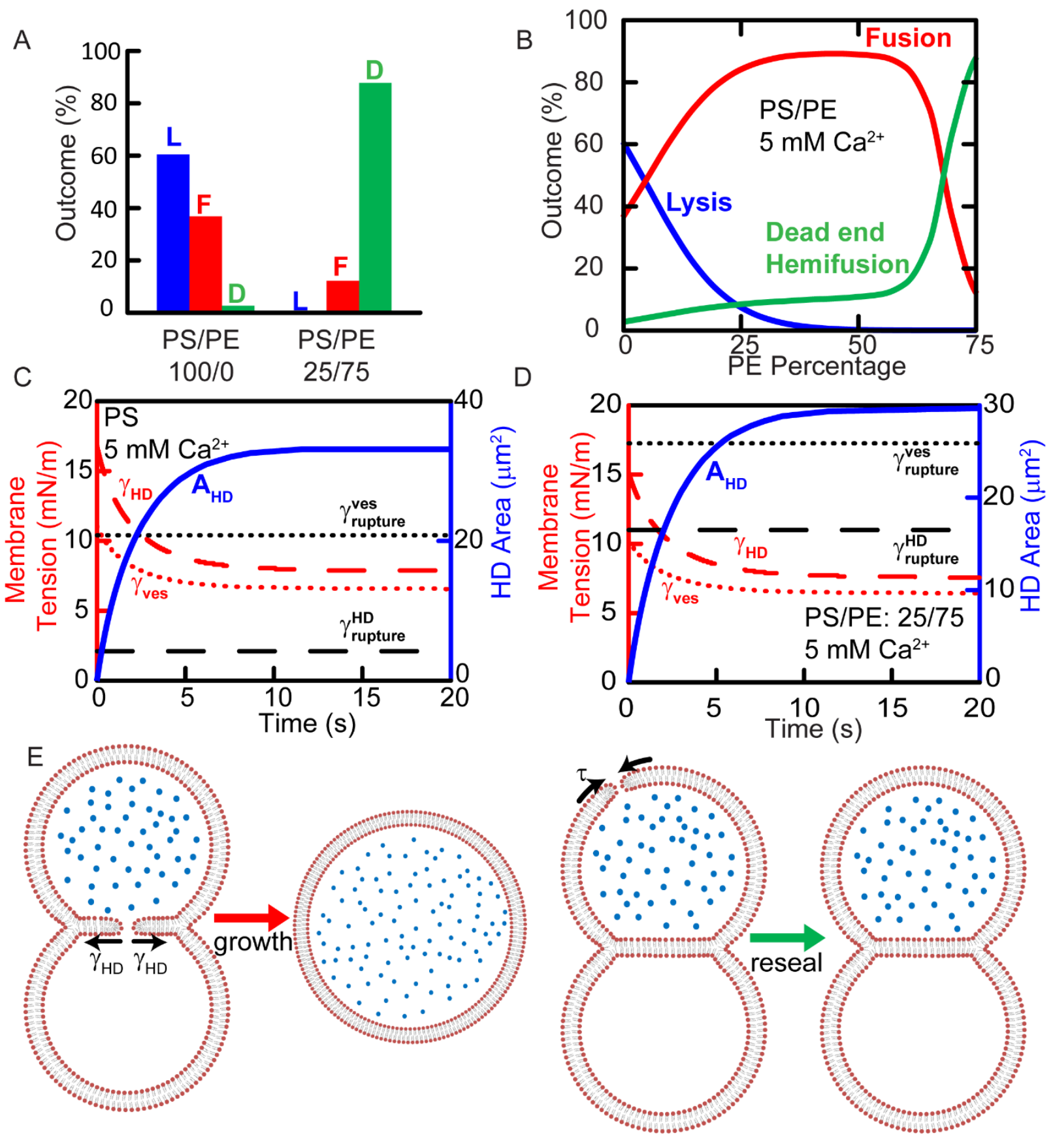
Figure 3- Lipid composition regulates outcome distribution in $\mathrm{Ca}^{2+}$-mediated GUV-GUV fusion. GUVs are made of PS and PE with different PE fractions. Model predictions, conditions as in experiments of refs. $(9,10)$ (see Table 1 for parameters). The negative curvature lipid PE disfavors pore formation and suppresses fusion. (A) Predicted outcome distributions for the compositions of ref. (9) (pure PS) and ref. (10) (75\% PE, 25\% PS) in the presence of $5 \mathrm{mM} \mathrm{Ca}^{2+}$. (B) Predicted outcome distributions versus PS/PE composition in the presence of $5 \mathrm{mM} \mathrm{Ca}{ }^{2+}$. $(\mathrm{C}$, D) Predicted time evolution of HD area, vesicle and HD tension for pure PS (C) and 75\% PE, $25 \%$ PS (D). (E) Mechanism that protects pure PS vesicles from lysis. As PS has positive spontaneous curvature nucleated pores have low line tension and will likely be expanded by the HD membrane tension $\gamma_{\mathrm{HD}}$ (left). By contrast the pore line tension $\tau$ in the vesicle membrane is large, as PS is thought to have negative spontaneous curvature in the presence of $\mathrm{Ca}^{2+}$, and pores close (right). Were it not for the PS curvature sign reversal, almost no fusion would occur as the pore nucleation rate is much greater in the far bigger vesicle membrane. 


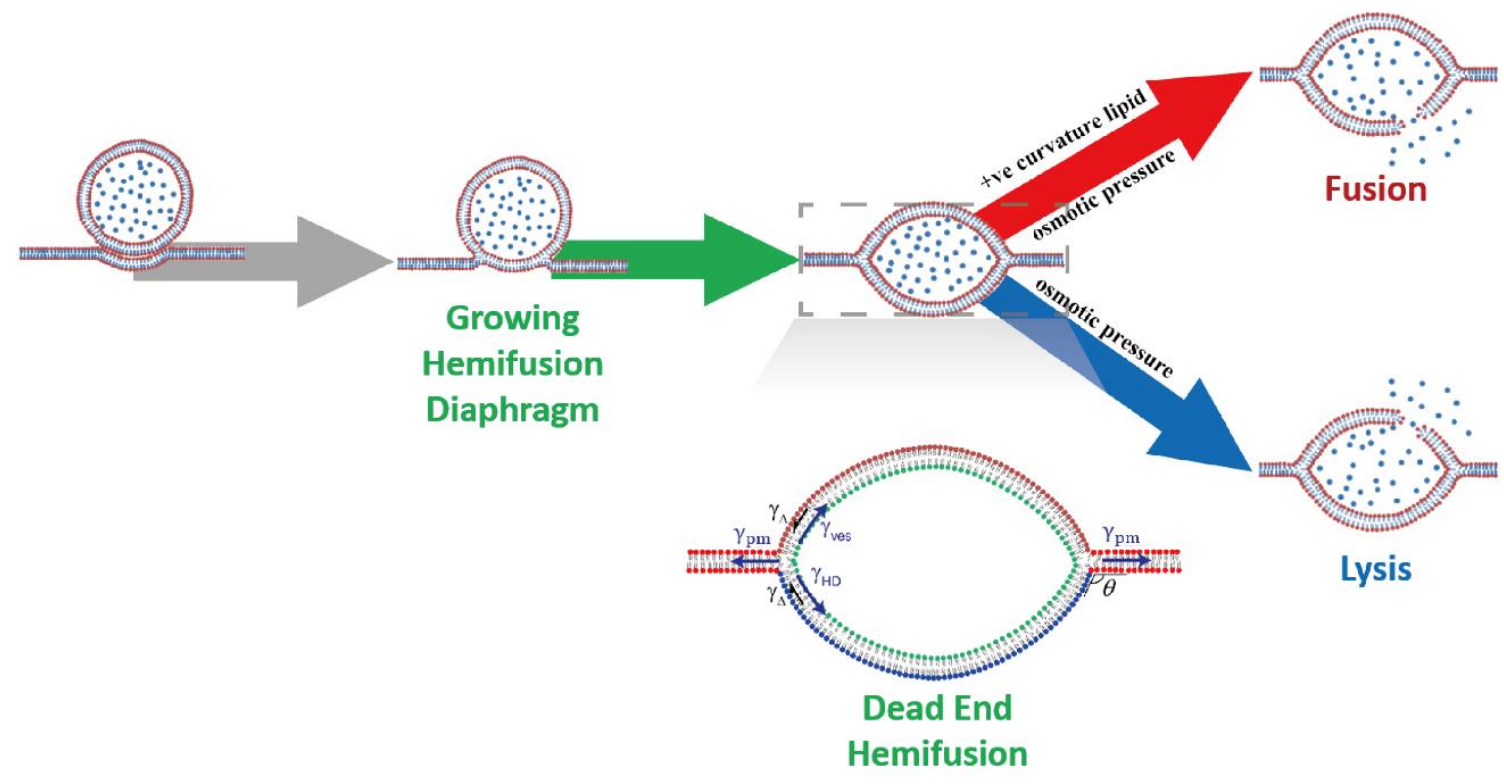

Figure 4- Calcium cannot fuse vesicles with planar membranes. Model predicted sequence is shown (schematic). At high [Ca2+], the vesicle has high tension and adheres strongly to the planar membrane, a PM in typical experiments. Following hemifusion and HD growth the present model predicts that vesicle tension is dissipated and a lens-shaped equilibrium hemifused state is reached. As the lens membrane tension is close to that of the PM, which is typically low, this is a dead-end state. Additional forces are required to drive fusion. Osmotically induced membrane tension will rupture either membrane with equal probability (fusion or lysis) while application of pore-promoting positive curvature lipids can selectively activate fusion or lysis. Zoom in: Equilibrium of vesicle fusing with a PM. The PM (light red lipid heads) is effectively a reservoir, and thus maintains a constant density and tension. The lipid density in the inner leaflet (green) decreases according to eq. S42. This density difference generates interleaflet tension $\gamma_{\Delta}$ which resists HD growth. The hemifused complex is symmetric about the PM, so the tension in the upper half of the hemifused region $\gamma_{\mathrm{ves}}$ is equal to the tension in the lower portion $\gamma_{\mathrm{HD}}$. At equilibrium, the force balance at the edge is given by eq. S44, which depends on the equilibrium contact angle $\theta$. 